\title{
Simulação de uma Pluma de Contaminantes no Rio Macaé Utilizando Redes Neurais Artificiais
}

\author{
Wagner Rambaldi Telles ${ }^{1}$, Pedro Paulo Gomes Watts Rodrigues ${ }^{1}$, Antônio José da Silva Neto ${ }^{1}$, \\ Orestes Llanes Santiago ${ }^{2}$, Mirtha Irizar Mesa ${ }^{2}$, Jader Lugon Junior ${ }^{3}$ \\ wtelles@iprj.uerj.br; pwatts@iprj.uerj.br; ajsneto@iprj.uerj.br; orestes@electrica.cujae.edu.cu; \\ mirtha@electrica.cujae.edu.cu; \\ jljunior@iff.edu.br
}

Recebido: 08/12/11 - revisado: 22/02/12 - aceito: 12/12/12

\begin{abstract}
RESUMO
O risco de falta d'água potável é um assunto abordado e debatido em todo o mundo. Alguns países já sofrem com esse problema devido à poluição descontrolada das águas através de resíduos industriais e domésticos, dentre outros. Baseado nisso, modelos matemáticos e computacionais têm sido constantemente utilizados no estudo do transporte de contaminantes em sistemas fluviais, tornando-se importantes na prevenção e estimativa do comportamento de plumas de contaminantes nesses corpos hídricos. Neste trabalho busca-se uma primeira abordagem para a modelagem do comportamento de contaminantes no Rio Macaé, um importante rio brasileiro situado no nordeste do Estado do Rio de Janeiro, tendo como ponto de partida estudos iniciais baseados em Redes Neurais Artificiais em substituição a um modelo matemático $2 D$ de transporte de poluentes. Pretende-se em um primeiro instante, simular o comportamento de uma pluma de contaminante em um trecho do Rio Macaé, confrontando os resultados experimentais obtidos em dois pontos do mesmo com os resultados numéricos obtidos via redes neurais treinadas pelo método de Levenberg-Marquardt. Os resultados apresentados neste trabalho servirão como base para um posterior treinamento de uma rede neural com o intuito de se obter a concentração em qualquer ponto do trecho de interesse do Rio Macaé.
\end{abstract}

Palavras-Chave: Rio Macaé, transporte de poluentes, redes neurais artificiais.

\section{INTRODUÇÃOO}

Um dos grandes desafios que se apresentam para a Humanidade neste século é o desenvolvimento sustentável. No que concerne a gestão de recursos hídricos, esse desenvolvimento deve ser entendido como aquele que maximize a produção e minimize os riscos ambientais. Atividades industriais necessariamente geram passivos ambientais, dentre os quais se destaca a diluição de efluentes como os mais graves. A preocupação com esse impacto ambiental está presente em diversos pontos da legislação ambiental

\footnotetext{
1 - Departamento de Engenharia Mecânica e Energia, Instituto Politécnico, UERJ

2 - Departamento de Automática e Computação, ISPJAE - Instituto Superior Politécnico José Antônio Echeverria

3 - Núcleo de Pesquisa em Petróleo, Energia e Recursos Naturais, IFF - Instituto Federal de Educação, Ciência e Tecnologia Fluminense
}

brasileira, mas de forma particular nas resoluções CONAMA que versam sobre a classificação e o enquadramento de corpos hídricos. Especificamente falando, a resolução 357/2005 impõe que estudos específicos sejam conduzidos na zona que compreenda a mistura de efluentes com as águas dos corpos receptores, sejam esses lagos, rios ou estuários. A avaliação do comportamento dessa diluição é complexa e operacionalmente custosa. Contudo, parte das dificuldades inerentes a esse diagnóstico pode ser contornada com a utilização de modelos que simulem o comportamento de poluentes uma vez lançados nesses corpos hídricos. Tais modelos podem ser vantajosos por diversos aspectos: a relação custo/benefício, a redução do tempo de análise, a agilização no processo de tomada de decisões, etc. Modelos matemáticos e computacionais se enquadram nessa categoria de ferramentas (TELLES, 2008).

Esses modelos são compostos por equações cuja solução fornece a distribuição no espaço e tempo de constituintes que sejam transportados 
pelo corpo hídrico em questão. Contudo, a formulação matemática de tais modelos muitas vezes inclui parâmetros, cuja estimativa pode ser crucial para a precisão da simulação que se deseje. Por exemplo, Shen et al. (2006) utilizou uma modificação do método de Newton para estimar fontes não pontuais de coliformes fecais, com o objetivo de estabelecer a carga máxima admissível para o Rio Wye. Por outro lado, Yang e Hamrick (2004) estimaram a salinidade na fronteira marinha de estuários através de problemas inversos. Já Strub et al. (2009) propuseram um novo algoritmo para estimar a condição de fronteira para modelos hidrodinâmicos aplicados em canais de maré. Por último, Rodrigues et al. (2012ab) estimaram parâmetros de dispersão em rios utilizando os métodos de Luus-Jaakola e Simulated Annealing.

Outra técnica que já apresentou resultados satisfatórios na modelagem de recursos hídricos em algumas aplicações são as redes neurais artificiais. Como exemplo, tem-se a caracterização do transporte hidrodinâmico no estuário do Rio Macaé (LIMA, 2012), a previsão de vazões naturais através da análise e tratamento de informações advindas de diferentes postos pluviométricos e fluviométricos na Bacia do Rio São Francisco (GOMES et al., 2010), a produção de energia em sistemas de abastecimento baseada em fontes renováveis (GONÇALVES et al., 2009), a criação de modelos de previsão de vazão considerando a chuva observada e/ou prevista (FIGUEIREDO et al., 2007) e a análise da elevação do nível de rios através da previsão das chuvas (DORNELLES et al., 2006). Além disso, com um correto treinamento da rede neural, é possível realizar a substituição do modelo matemático pela mesma, evitando a necessidade de se estimar determinados parâmetros.

Neste trabalho são discutidas e aplicadas técnicas muitas vezes adotadas na simulação do comportamento de plumas de poluentes em rios. As técnicas foram empregadas em um estudo de caso, que consistiu no lançamento de traçador conservativo (cloreto de sódio - $\mathrm{NaCl}$ ) em trecho do baixo curso do Rio Macaé, situado na costa norte do estado do Rio de Janeiro (COSTA, 1999). Considerando dois pontos de coleta, a evolução no tempo das concentrações do traçador foi comparada, entre os valores gerados pela solução de um modelo de transporte 2D (integrado na vertical) e aqueles gerados a partir da aplicação de redes neurais artificiais, essas com uma arquitetura baseada no perceptron de múltiplas camadas (MLP) com retro-propagação de erro (backpropagation) (HAYKIN, 1994), com treinamento pelo método de Levenberg-Marquardt.

\section{ESTUDO DE CASO - RIO MACAÉ}

Como salientado, esse estudo incluiu um ensaio experimental conduzido no baixo Macaé, que representa o mais importante rio estritamente estadual que drena o território fluminense. Esse rio possui uma área de drenagem de aproximadamente $1.765 \mathrm{~km}^{2}$, sendo que sua bacia apresenta ocupação bastante heterogênea, desde áreas de preservação a importantes núcleos urbanos, a se destacar a cidade de Macaé, que se situa às margens de seu baixo curso e na qual se concentram importantes atividades ligadas à indústria do petróleo. A Figura 1 apresenta a localização da área onde se deu o ensaio.

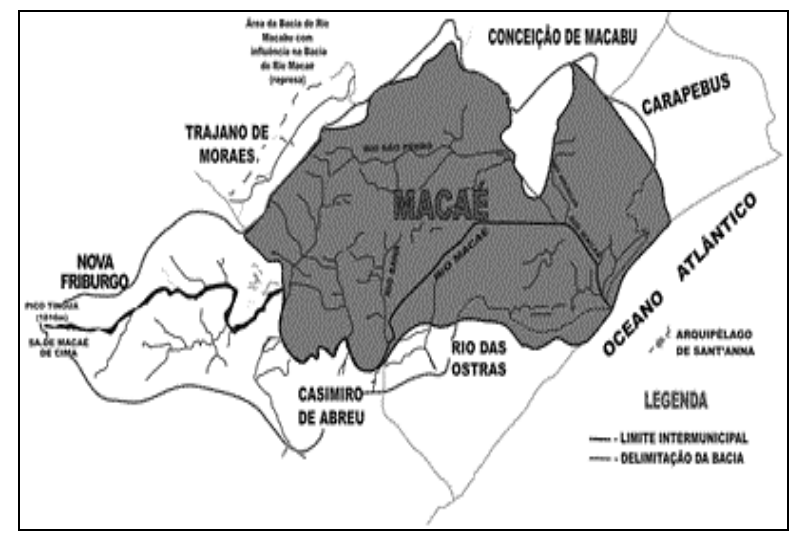

Figura 1 - Mapa do Rio Macaé onde está localizada a região de interesse ${ }^{2}$.

\section{Ensaio Experimental}

O trabalho de campo que deu suporte a modelagem apresentada neste artigo foi realizado em um trecho retilinizado do baixo Rio Macaé. Nessa região o rio apresenta profundidade média em torno de $1 \mathrm{~m}$ e largura praticamente uniforme de 40 m. No cenário aqui simulado (vazão de estio) as velocidades médias não excedem $1 \mathrm{~m} / \mathrm{s}$ (AMARAL, 2003).

Para a simulação do comportamento de uma pluma de contaminante na região estudada, foi utilizado $\mathrm{NaCl}$ como traçador, escolhido devido a sua baixa toxicidade e fácil manipulação (RODRIGUES, et al., 2012a). Para a preparação do traçador, diluiu-se $2000 \mathrm{~g}$ de $\mathrm{NaCl}$ em água coletada do próprio rio, resultando em $11 \mathrm{~L}$ de solução assim pre-

${ }^{2}$ Fonte: http://www.macae.rj.gov.br/semaph/riomacae 
parada. Esse procedimento se repetiu por dez vezes a fim de se gerar dois recipientes contendo $55 \mathrm{~L}$ de solução. Por meio de um condutivímetro e através da curva de calibração (condutividade versus concentração) do mesmo, determinou-se uma concentração de $1,75 \times 10^{5} \mathrm{mg} / \mathrm{L}$ para essa solução, contrastando com uma concentração de $37 \mathrm{mg} / \mathrm{L}$ determinada na água do rio.

O experimento foi realizado ao final do mês de maio de 2008, no período em que Rio Macaé apresenta menores vazões, em torno de $6 \mathrm{~m}^{3} / \mathrm{s}$ (AMARAL, 2003), estando, portanto, mais sensível à diluição de efluentes(LUGON et al., 2008).

O lançamento da solução de traçador se deu de forma instantânea, ocorrendo a $0,50 \mathrm{~m}$, sentido transversal, da margem do rio. Já as coletas das amostras ocorreram em dois pontos situados $50 \mathrm{~m}$ a jusante do ponto de lançamento, o primeiro a 0,50 $\mathrm{m}$ e o segundo a 3,00 $\mathrm{m}$ da margem. Amostras de água foram coletadas a intervalos regulares de $12 \mathrm{~s}$. Todo o experimento foi conduzido em um intervalo de tempo de $360 \mathrm{~s}$.

\section{MODELO MATEMÁTICO DE TRANSPORTE 2D}

A solução do problema direto consistiu na simulação da evolução espaço-temporal do transporte de substância conservativa injetada de forma instantânea no domínio, no caso um trecho do baixo Macé.. Para tanto, implementou-se a solução numérica de um modelo de transporte 2D (integrado na vertical), desprezando-se a componente transversal do termo advectivo, cuja equação é dada por (TELLES, 2009):

$$
\frac{\partial \mathrm{C}}{\partial \mathrm{t}}+\mathrm{u} \frac{\partial \mathrm{C}}{\partial \mathrm{x}}=\mathrm{E}_{1} \frac{\partial}{\partial \mathrm{x}}\left(\frac{\partial \mathrm{C}}{\partial \mathrm{x}}\right)+\mathrm{E}_{\mathrm{t}} \frac{\partial}{\partial \mathrm{y}}\left(\frac{\partial \mathrm{C}}{\partial \mathrm{y}}\right)
$$

com as seguintes condições de contorno e inicial:

$$
\begin{aligned}
& \mathrm{C}(0, \mathrm{y}, \mathrm{t})=\mathrm{C}_{0}, 0 \leq \mathrm{y} \leq \mathrm{L}_{\mathrm{y}}, \mathrm{t}>0 \\
& \frac{\partial \mathrm{C}\left(\mathrm{L}_{\mathrm{x}}, \mathrm{y}, \mathrm{t}\right)}{\partial \mathrm{x}}=0,0 \leq \mathrm{y} \leq \mathrm{L}_{\mathrm{y}}, \mathrm{t}>0
\end{aligned}
$$

$\frac{\partial \mathrm{C}(\mathrm{x}, 0, \mathrm{t})}{\partial \mathrm{y}}=\frac{\partial \mathrm{C}\left(\mathrm{x}, \mathrm{L}_{\mathrm{y}}, \mathrm{t}\right)}{\partial \mathrm{y}}=0,0 \leq \mathrm{x} \leq \mathrm{L}_{\mathrm{x}}, \mathrm{t}>0$

$\mathrm{C}(\mathrm{x}, \mathrm{y}, 0)=\mathrm{C}_{1}(\mathrm{x}, \mathrm{y}), 0 \leq \mathrm{x} \leq \mathrm{L}_{\mathrm{x}}, 0 \leq \mathrm{y} \leq \mathrm{L}_{\mathrm{y}}$

onde:

$\mathrm{C}=$ concentração $[\mathrm{mg} / \mathrm{l}]$

$\mathrm{t}=$ tempo $[\mathrm{s}]$

$\mathrm{x}, \mathrm{y}=$ domínio espacial $[\mathrm{m}]$;

$\mathrm{u}=$ velocidade $[\mathrm{m} / \mathrm{s}]$;

$\mathrm{E}_{1}, \mathrm{E}_{\mathrm{t}}=$ coeficientes de difusão da concentração nas direções $\mathrm{x}, \mathrm{y}$, respectivamente $\left[\mathrm{m}^{2} / \mathrm{s}\right]$;

$\mathrm{L}_{\mathrm{x}}=$ comprimento longitudinal do trecho do rio em análise $[\mathrm{m}]$;

$\mathrm{L}_{\mathrm{y}}=$ comprimento transversal do trecho do rio em análise $[\mathrm{m}]$.

Devido à geometria bastante regular do rio no trecho simulado, pode-se assumir velocidades longitudinais uniformes no sentido transversal e desprezar as próprias componentes transversais, o que simplificou consideravelmente o problema a ser resolvido. Dessa forma, os parâmetros mais relevantes no trecho em análise são os coeficientes de dispersão longitudinal e transversal.

A Equação (1) foi resolvida numericamente utilizando-se o método de Volumes Finitos, baseado em uma formulação implícita e aproximação do termo advectivo através do esquema UpWind (UDS), como mostrado na Equação (3) (TELLES, 2009),

$$
\begin{aligned}
& \left(-\mathrm{E}_{\mathrm{t}} \frac{\Delta \mathrm{x}}{\Delta \mathrm{y}}\right) \mathrm{C}_{\mathrm{S}}^{\mathrm{t}+\Delta \mathrm{t}}+\left(-\mathrm{u} \Delta \mathrm{y}-\mathrm{E}_{1} \frac{\Delta \mathrm{y}}{\Delta \mathrm{x}}\right) \mathrm{C}_{\mathrm{w}}^{\mathrm{t}+\Delta \mathrm{t}}+ \\
& \left(\frac{\Delta \mathrm{x} \Delta \mathrm{y}}{\Delta \mathrm{t}}+\mathrm{u} \Delta \mathrm{y}+2 \mathrm{E}_{1} \frac{\Delta \mathrm{y}}{\Delta \mathrm{x}}+2 \mathrm{E}_{\mathrm{t}} \frac{\Delta \mathrm{x}}{\Delta \mathrm{y}}\right) \mathrm{C}_{\mathrm{P}}^{\mathrm{t}+\Delta \mathrm{t}}+ \\
& \left(-\mathrm{E}_{1} \frac{\Delta \mathrm{y}}{\Delta \mathrm{x}}\right) \mathrm{C}_{\mathrm{E}}^{\mathrm{t}+\Delta \mathrm{t}}+\left(-\mathrm{E}_{\mathrm{t}} \frac{\Delta \mathrm{x}}{\Delta \mathrm{y}}\right) \mathrm{C}_{\mathrm{N}}^{\mathrm{t}+\Delta \mathrm{t}}=\frac{\Delta \mathrm{x} \Delta \mathrm{y}}{\Delta \mathrm{t}} \mathrm{C}_{\mathrm{P}}^{\mathrm{t}}
\end{aligned}
$$

Essa equação gera um sistema da forma $A x=b$, onde $A$ é uma matriz pentadiagonal, sendo então resolvido pelo Algoritmo de Thomas (TDMA). Toda a implementação foi realizada na linguagem de programação C. 


\section{REDES NEURAIS ARTIFICIAIS}

As Redes Neurais Artificiais (RNAs) são estruturas paralelas compostas de neurônios artificiais - os quais simulam os neurônios biológicos humanos - densamente conectados que apresentam duas fases distintas de operação: a aprendizagem (treinamento) e a generalização.

O neurônio artificial é uma estrutura lógicomatemática que procura simular a forma, o comportamento e a funcionalidade de um neurônio biológico. Dessa forma, os dendritos são substituídos por entradas cujas ligações com o corpo celular artificial são realizadas através de elementos chamados de pesos, os quais simulam as sinapses. Os estímulos captados pelas entradas são processados pela função soma e o limiar de disparo do neurônio biológico é representado pela função transferência (TONSIG, 2000). A maioria dos modelos de redes neurais possui alguma regra de treinamento, onde os pesos e suas conexões são ajustados com os padrões apresentados; elas aprendem através de exemplos (YAMAMOTO e NIKIFORUK, 2000). Dentre as diferentes topologias neurais considera-se que as redes perceptron de múltiplas camadas (MLP), que usam em seu treinamento a retro-propagação do erro, são aquelas utilizadas com maior frequência devido à facilidade de implementação e sintaxe menos robusta.

Com o intuito de modelar o comportamento da concentração na região de interesse, buscando-se simular, através da substituição do modelo de transporte 2D integrado na vertical, os dados obtidos no trabalho de campo descrito anteriormente, foi realizado o treinamento da rede utilizando o algoritmo backpropagation e o método de LevenbergMarquardt para a determinação dos pesos e bias, o qual, segundo Kinoshita et al. (2000), aumenta a velocidade de treinamento sem perda de desempenho da rede.

O método de Levenberg-Marquardt é um método de otimização de segunda ordem o qual utiliza uma aproximação para a matriz Hessiana e para o gradiente a fim de atualizar os pesos e bias, ou seja (BIONDI NETO et al., 2006),

$$
\mathrm{H} \approx \mathrm{J}^{\mathrm{T}} \mathbf{J}
$$

onde $\mathrm{H}$ é a matriz Hessiana, J é a matriz Jacobiana que contém a derivada primeira dos erros da rede com relação aos pesos e bias.
O método de Levenberg-Marquardt procura encontrar o melhor ajuste para um conjunto de dados tentando minimizar a soma dos quadrados dos resíduos, obtendo um novo vetor de pesos $\mathrm{W}_{\mathrm{n}+1}$, partindo do vetor de pesos $\mathrm{W}_{\mathrm{n}}$, ou seja:

$\mathrm{w}_{\mathrm{n}+1}=\mathrm{w}_{\mathrm{n}}+\Delta \mathrm{w}_{\mathrm{n}}$

onde:

$\Delta \mathrm{w}_{\mathrm{n}}=-\left[\mathrm{J}_{\mathrm{n}}^{\mathrm{T}} \mathrm{J}_{\mathrm{n}}+\mu \mathrm{I}\right]^{-1} \mathrm{~J}_{\mathrm{n}}^{\mathrm{T}} \mathrm{e}(\mathrm{w})$

sendo e(w) a função erro, $\mu$ o parâmetro (fator de amortecimento) de Marquardt e I a matriz identidade.

Devido à aleatoriedade na escolha da primeira estimativa dos pesos e bias, cada simulação realizada neste trabalho foi processada em torno de 30 vezes a fim de se encontrar os melhores resultados numéricos, tendo como referência os dados experimentais obtidos no trabalho de campo.

Em todos os testes realizados, foram passadas como padrão de treinamento 44 informações referentes a 6 parâmetros de influência significativa na modelagem da pluma de contaminante, são eles: posição de lançamento na longitudinal, posição de lançamento na transversal, quantidade do traçador lançada no rio, posição de coleta na longitudinal, posição de coleta na transversal e tempo de coleta.

De posse dessas informações a rede pode ser caracterizada por uma matriz $\mathrm{P}_{6 \times 44}$ como padrão de entrada, a qual mapeia um vetor $\mathrm{T}_{1 \times 44}$ que representa as concentrações oriundas do trabalho de campo. A Figura 2 apresenta a estrutura dos dados de entrada e informações de saída utilizadas no treinamento da rede neural.

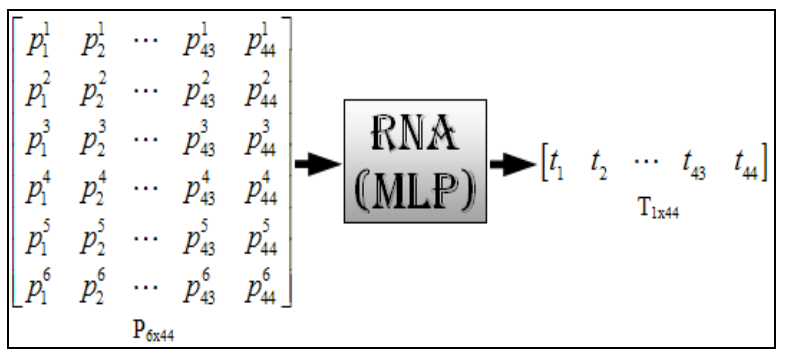

Figura 2 - Esquema da rede neural utilizada na simulação da pluma de contaminante no Rio Macaé. 
Para efeito de validação do treinamento, a rede foi testada com um conjunto de dados que não fizeram parte do treinamento.

A implementação do método proposto nesta seção foi realizada no programa Matlabß, assim como a construção dos gráficos referentes às simulações.

\section{RESULTADOS E DISCUSSÃO}

Foram realizadas comparações entre os resultados experimentais e as simulações obtidas através do modelo matemático $2 \mathrm{D}$ resolvido numericamente pelo método de volumes finitos e o treinamento de uma rede neural com minimização do erro médio quadrático por Levenberg-Marquardt. Os resultados numéricos foram gerados em um computador com processador Intel Core 2 Duo com processador de 1,90 GHz 2 GB de memória RAM. Em todas as simulações apresentadas aqui envolvendo o modelo matemático 2D, o tempo de execução não ultrapassou a 2 minutos, enquanto que o treinamento da rede neural foi inferior a 1 minuto.

$\mathrm{Na}$ Tabela 1 são mostrados os valores de "concentração versus tempo" utilizados nas simulações.

Tabela 1 - Valores das concentrações nas amostras utilizadas no treinamento da rede neural. $[\mathrm{C}]=\mathrm{mg} / \mathrm{l}$

\begin{tabular}{llll|ll}
\hline \multicolumn{3}{l}{ Concentração coletada } & & \multicolumn{2}{l}{$\mathbf{3 , 0}$ m da margem } \\
\hline $\mathbf{0 , 5}$ m da & margem & & & t(s) & $\mathbf{C}$ \\
\hline $\mathbf{t ( s )}$ & $\mathbf{C}$ & $\mathbf{t}(\mathbf{s})$ & $\mathbf{C}$ & $\mathbf{0}$ & 37,00 \\
\hline $\mathbf{0}$ & 37,00 & $\mathbf{2 1 4}$ & 112,84 & $\mathbf{2}$ & 44,97 \\
$\mathbf{1 2}$ & 40,29 & $\mathbf{2 2 2}$ & 108,82 & $\mathbf{1 0 0}$ & 111,93 \\
$\mathbf{2 4}$ & 38,16 & $\mathbf{2 3 0}$ & 102,39 & $\mathbf{1 0 8}$ & 130,01 \\
$\mathbf{3 5}$ & 39,48 & $\mathbf{2 4 0}$ & 93,22 & $\mathbf{1 1 6}$ & 179,56 \\
$\mathbf{4 2}$ & 38,48 & $\mathbf{2 5 8}$ & 82,11 & $\mathbf{1 2 4}$ & 171,26 \\
$\mathbf{4 9}$ & 37,99 & $\mathbf{2 7 5}$ & 70,16 & $\mathbf{1 3 6}$ & 25,02 \\
$\mathbf{7 5}$ & 39,77 & $\mathbf{2 8 0}$ & 67,34 & $\mathbf{1 8 0}$ & 144,02 \\
$\mathbf{8 6}$ & 41,67 & $\mathbf{3 0 0}$ & 56,56 & $\mathbf{2 1 2}$ & 110,75 \\
$\mathbf{9 2}$ & 41,34 & $\mathbf{3 0 7}$ & 57,63 & $\mathbf{2 6 6}$ & 79,04 \\
$\mathbf{1 0 0}$ & 126,88 & $\mathbf{3 1 2}$ & 57,36 & $\mathbf{3 1 4}$ & 55,58 \\
$\mathbf{1 0 9}$ & 196,70 & $\mathbf{3 2 0}$ & 56,56 & $\mathbf{3 4 2}$ & 47,01 \\
$\mathbf{1 3 6}$ & 363,12 & $\mathbf{3 2 8}$ & 52,03 & & \\
$\mathbf{1 4 6}$ & 230,86 & $\mathbf{3 3 5}$ & 47,34 & & \\
$\mathbf{1 7 0}$ & 187,94 & $\mathbf{3 4 3}$ & 47,54 & & \\
$\mathbf{1 9 0}$ & 157,66 & $\mathbf{3 4 8}$ & 45,88 & & \\
$\mathbf{1 9 4}$ & 150,78 & $\mathbf{3 5 2}$ & 47,14 & & \\
$\mathbf{2 0 5}$ & 123,43 & & & & \\
\hline
\end{tabular}

Com base nas concentrações experimentais foi possível estimar o perfil da concentração nos dois pontos de coleta para os demais instantes de tempos onde não ocorreram medições. A Figura 3 mostra ajuste dos dados por meio de uma interpolação linear.

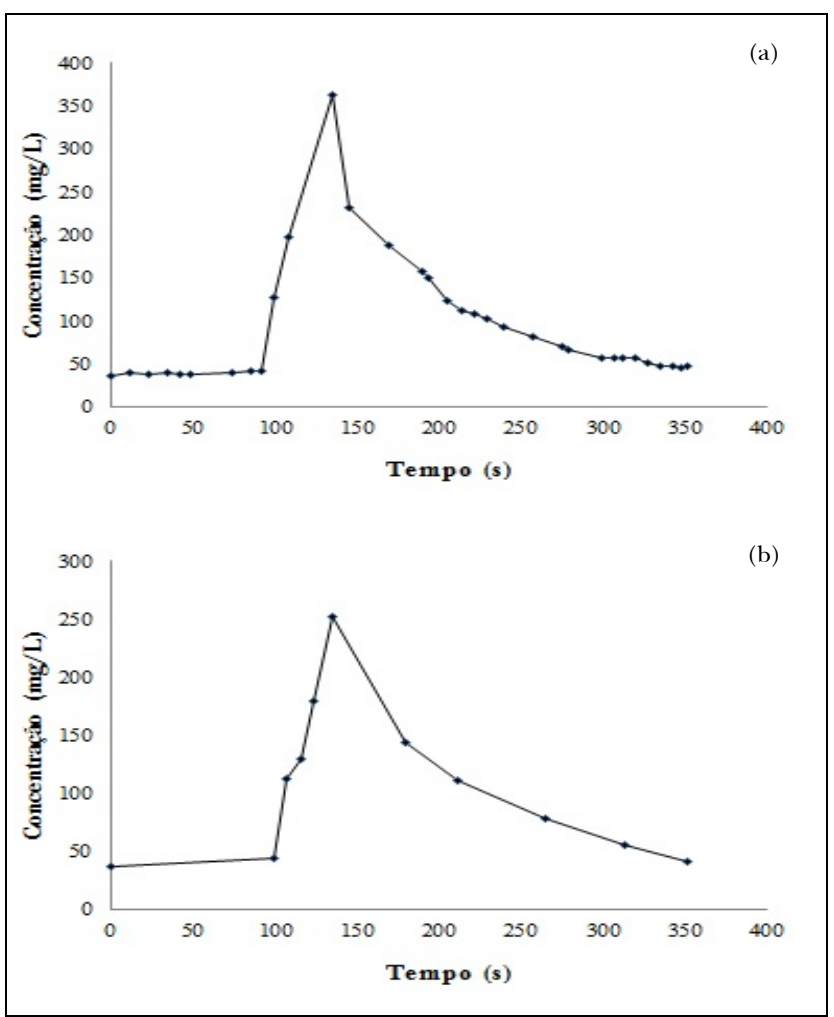

Figura 3 - (a) Concentração a 0,5 m da margem do rio; (b) Concentração a 3,0 m da margem do rio.

Tabela 2 - Valores dos parâmetros utilizados na simulação.

\begin{tabular}{ll}
\hline Parâmetros & Valores \\
\hline Comprimento longitudinal [m] & 182,00 \\
Comprimento transversal [m] & 40,00 \\
Posição de lançamento em x [m] & 50,00 \\
Posição de lançamento em y [m] & 0,50 \\
Posição de coleta em x [m] & 100,00 \\
Posição de coleta em y [m] & 0,50 \\
$\Delta[\mathrm{s}]$ & 2,00 \\
$\Delta \mathrm{x}[\mathrm{m}]$ & 0,70 \\
$\Delta \mathrm{y}[\mathrm{m}]$ & 0,70 \\
Velocidade [m/s] & 0,35 \\
$\mathrm{E}_{\mathrm{l}}\left[\mathrm{m}^{2} / \mathrm{s}\right]$ & 0,033 \\
$\mathrm{E}_{\mathrm{t}}\left[\mathrm{m}^{2} / \mathrm{s}\right]$ & 0,008 \\
\hline
\end{tabular}




\section{Solução utilizando o Modelo Matemático}

Foram realizados alguns testes com o objetivo de se determinar a melhor configuração dos parâmetros necessários à simulação envolvendo o modelo matemático, os quais são mostrados na Tabela 2.

A Figura 4 apresenta os resultados obtidos com os parâmetros descritos na Tabela 2 .

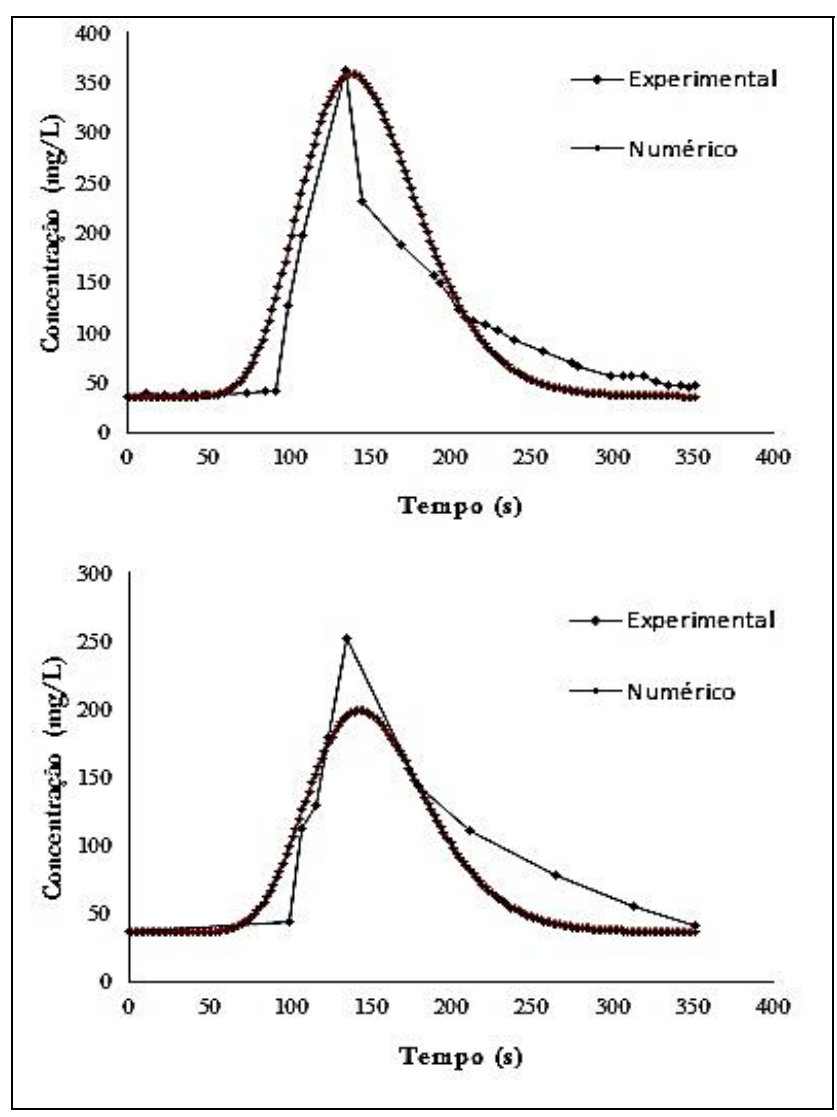

Figura 4 - (a) Concentrações numéricas e experimentais a 0,5 m da margem do rio; (b) Concentrações numéricas e experimentais a $3,0 \mathrm{~m}$ da margem do rio.

Como verificado na Figura 3(b), não houve um bom ajuste entre o modelo matemático e os dados experimentais. Isso se deve ao fato da dificuldade de calibração dos parâmetros, como já mencionado anteriormente. Devido a isso, é proposta a substituição do modelo matemático por redes neurais artificiais.

\section{Solução utilizando Redes Neurais}

Foram realizadas simulações variando o número de camadas ocultas (de uma a cinco cama- das) da rede neural, bem como o número de neurônios (de 5 a 50 neurônios) presentes em cada camada, a fim de se obter o melhor ajuste entre os resultados numéricos e os dados experimentais.

Para o treinamento da rede as posições de lançamento na longitudinal e na transversal foram definidas como $50,0 \mathrm{~m}$ e $0,50 \mathrm{~m}$, respectivamente, enquanto que a concentração inicial lançada instantaneamente no rio como $175 \mathrm{~g} / \mathrm{L}$. Já as posições de coleta das amostras foram de $100 \mathrm{~m}$ na longitudinal e $0,50 \mathrm{~m}$ na transversal para o primeiro ponto e 100 $\mathrm{m}$ na longitudinal e $3 \mathrm{~m}$ na transversal para o segundo ponto. Os instantes de tempo nos quais se deram as coletas estão listados na Tabela 1.

No que se refere à validação, foi verificado o comportamento da rede a cada 60 s dentro do intervalo $[0,360]$ segundos, para os dois pontos de coleta.

Inicialmente foi realizado o treinamento da rede neural utilizando apenas uma camada oculta e variando a quantidade de neurônios nesta camada. A Tabela 3 contém os parâmetros, enquanto a Figura 5 apresenta os resultados obtidos com a melhor configuração para o treinamento da rede com uma camada oculta.

Tabela 3 - Valores da configuração da rede neural com uma camada oculta utilizada nas simulações.

\begin{tabular}{c|c}
\hline Parâmetros & Valores \\
\hline $\begin{array}{c}\text { Número de neurônios } \\
\text { na camada oculta }\end{array}$ & 30 \\
\hline Funções de ativação & $\begin{array}{c}\text { Tangente } \\
\text { Hiperbólica } \\
\text { Linear }\end{array}$ \\
\hline Método & Levenberg- \\
Marquardt
\end{tabular}

Verifica-se que o treinamento da rede neural com apenas uma camada oculta não conseguiu modelar satisfatoriamente o pico de concentração, como visto na Figura 5 (c) e o início da pluma, Figura 5 (d), obtendo a raiz do erro médio quadrático (RMSE) de aproximadamente $22 \mathrm{mg} / \mathrm{L}$. Foi realizado então o treinamento da rede neural utilizando duas camadas ocultas e variando o número de neurônios das mesmas a fim de se obter o melhor conjunto de parâmetros que representasse numericamente os dados obtidos no trabalho de campo. A validação se deu com o mesmo conjunto de dados utilizados para o teste da rede com apenas uma ca- 

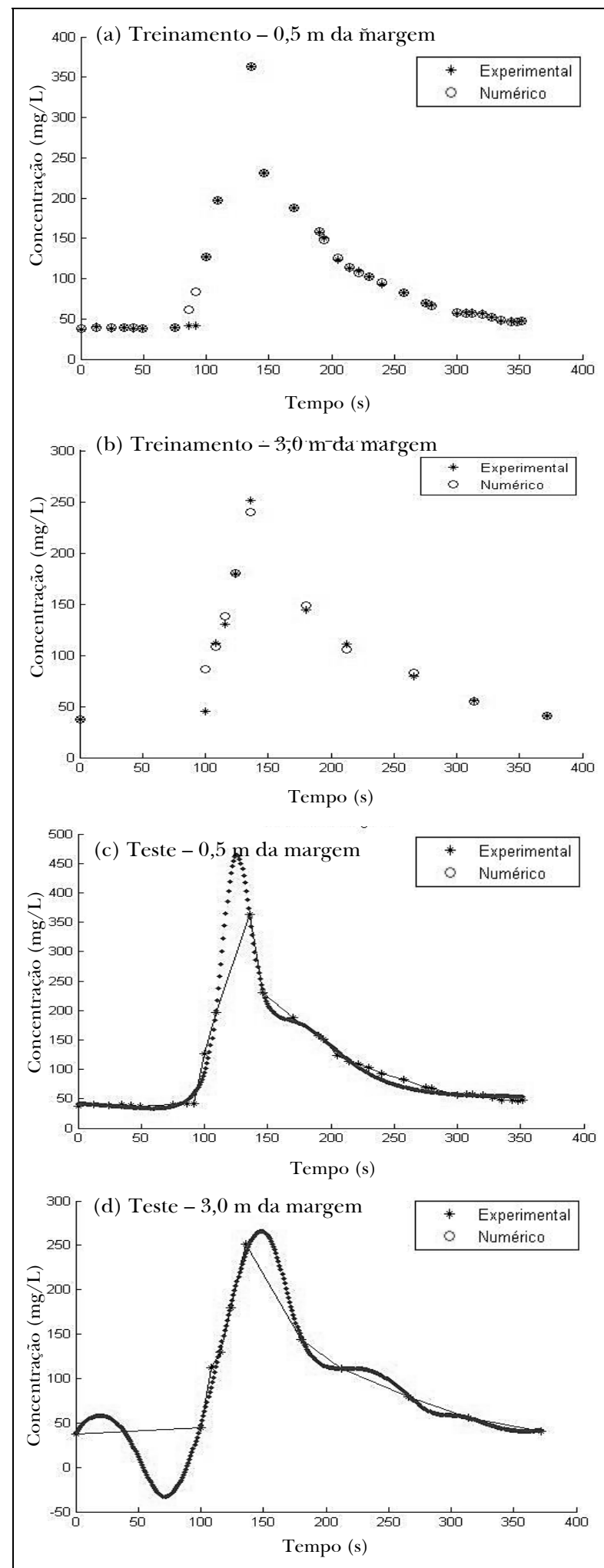

Figura 5 - Concentrações obtidas nos pontos de coleta a $0,5 \mathrm{~m}$ da margem após o treinamento e teste da rede neural com 30 neurônios na camada escondida, após 531 épocas.
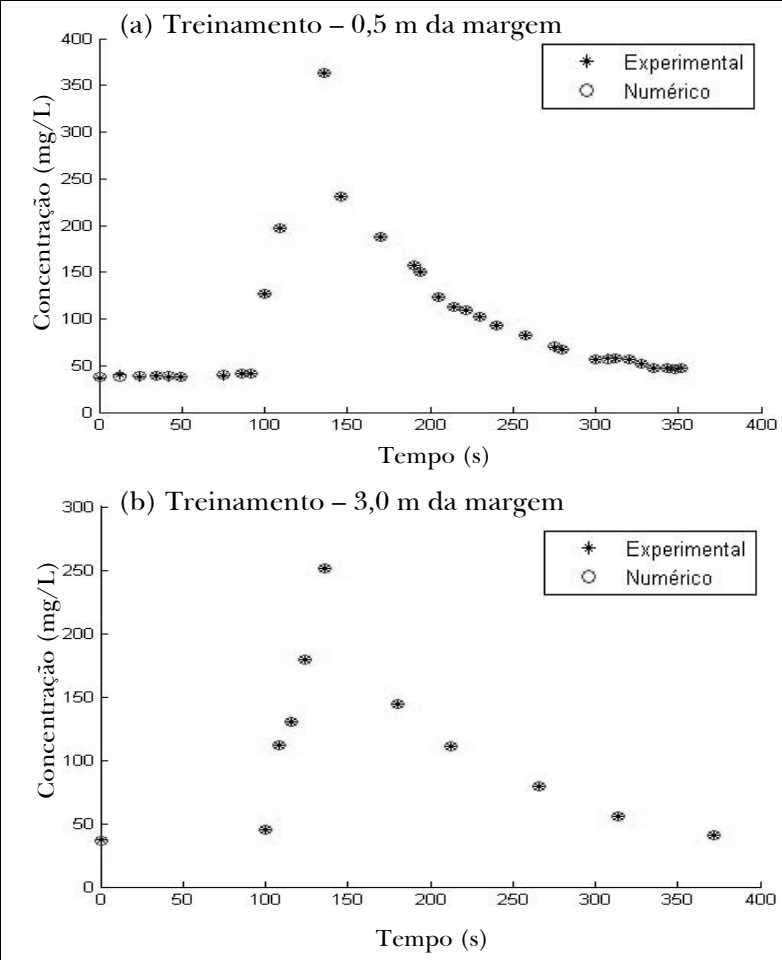

(c) Teste $-0,5 \mathrm{~m}$ da margem

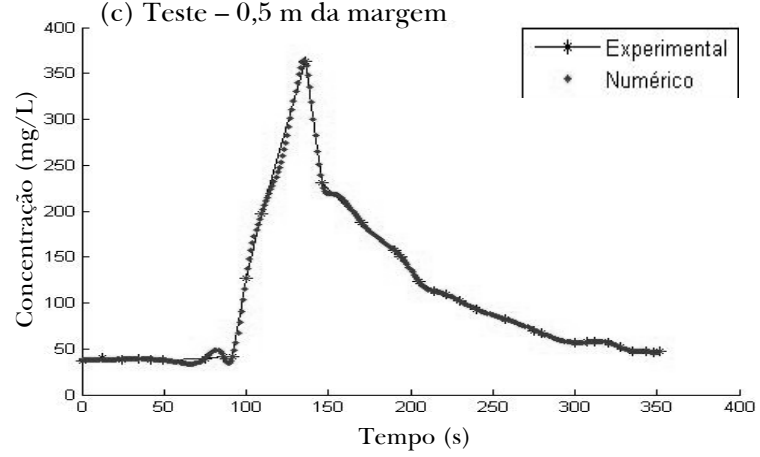

(d) Teste $-3,0 \mathrm{~m}$ da margem

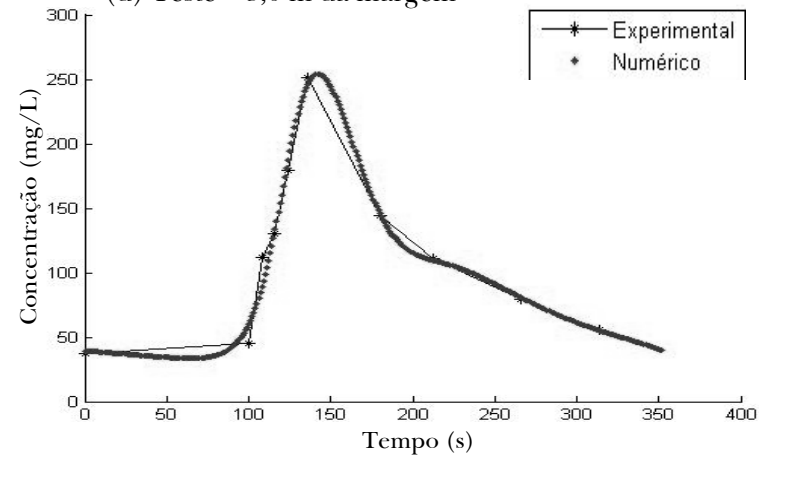

Figura 6 - Concentrações obtidas nos pontos de coleta a 0,5 m da margem após o treinamento e teste da rede neural com 14 e 8 neurônios na primeira e segunda camada escondida, respectivamente, após 1280 épocas. 
mada oculta. A Figura 6 apresenta os resultados obtidos com o treinamento da rede com a melhor configuração, contendo 14 e 8 neurônios na primeira e na segunda camada escondida, respectivamente.

As funções de ativação foram Tangente Hiperbólica, Tangente Hiperbólica e Linear. Os demais parâmetros são os mesmos apresentados na Tabela 3 .

Observa-se na Figura 6, um melhor ajuste do resultado numérico aos dados experimentais no que se refere ao pico de concentração da pluma de contaminante (traçador), tendo atingido a raiz do erro médio quadrático em torno de $1,7 \mathrm{mg} / \mathrm{L}$.

Outros testes foram realizados com a rede contendo três, quatro e cinco camadas ocultas. No entanto, os resultados mostraram-se inferiores ao treinamento com duas camadas ocultas.

\section{CONCLUSÕES E CONSIDERAÇÕES}

O objetivo desse trabalho foi realizar uma primeira abordagem para a modelagem do comportamento de contaminantes no Rio Macaé, tendo como ponto de partida estudos iniciais baseados em Redes Neurais Artificiais em substituição a um modelo matemático 2D de transporte de poluentes. Verifica-se que o treinamento da rede com uma e duas camadas ocultas apresentou uma boa correlação frente aos valores utilizados para essa finalidade. No entanto, ao se utilizar dados que não fizeram parte do treinamento, conclui-se que a rede com apenas uma camada oculta não conseguiu modelar de forma satisfatória o pico de concentração da pluma de contaminante, como mostra a Figura 5, diferentemente do treinamento utilizando duas camadas ocultas, o qual conseguiu um melhor ajuste numérico em relação aos referidos dados, como mostra a Figura 6. Isso mostra mostra que a rede treinada com apenas uma camada oculta não foi capaz de realizar uma generalização para a modelagem da pluma de contaminante quando utilizado um conjunto de padrões que não fizeram parte de seu treinamento.

A partir deste estudo inicial, cuja principal vantagem se deve ao fato da rede neural não necessitar estimar os parâmetros de transporte, como no modelo matemático, busca-se, como expectativa para trabalhos futuros, uma generalização da rede neural com o intuito de se obter a concentração em qualquer ponto do rio no trecho de interesse e em qualquer instante de tempo. Ressalta-se, porém, que a principal dificuldade nesse processo está relacionada com o treinamento da rede, que exige grande quantidade de dados experimentais, cuja obtenção pode esbarrar em limitações logísticas e operacionais.

\section{AGRADECIMENTOS}

Os autores agradecem à CAPES, CPNq, FAPERJ e à empresa Termomacaé Ltda., bem como aos revisores cujos comentários permitiram melhorar o conteúdo do trabalho.

\section{REFERÊNCIAS}

AMARAL, K. (2003). Estuário do rio macaé: Modelagem computacional como ferramenta para o gerenciamento integrado de recursos hídricos. Dissertação (Mestrado em Engenharia Civil), COPPE/UFRJ. Rio de Janeiro.

BIONDI NETO, L.; SIEIRA, A.C.C.F.; DANZIGER, B.R.; SILVA, J.G.S. (2006). Classificação de Solos usando-se Redes Neurais Artificiais. Engevista (UFF), v. 08, p. 37-48.

COSTA, H. (1999). Subsídios para Gestão dos Recursos Hidricos das Bacias Hidrográficas dos Rios Macacu, São João, Macaé e Macabu. Secretaria de Estado de Meio Ambiente e Desenvolvimento Sustentável (SEMADS), Rio de Janeiro.

DORNELLES, F.; PEDROLLO, O.C.; GOLDENFUM, J.A. (2006). Análise comparativa da aplicação de redes neurais e sistemas de inferência difusa na previsão de nível do rio Quaraí, utilizando previsão de chuva. In: Anais do Simpósio Brasileiro de Recursos Hídricos do Sul-Sudeste, Curitiba, v. 1.

FIGUEIREDO， K.; BARBOSA， C.R.H.; CRUZ, A.V.A.; VELLASCO, M.; PACHECO, M.A.C.; CONTRERAS, R.J.; BARROS, M.; SOUZA, R.C.; MARQUES, V.S.; DUARTE, U.M. (2007). Modelo de previsão de vazão com informação de precipitação utilizando Redes Neurais. Revista Brasileira de Recursos Hídricos, v. 12, n. 3, p. 69-82.

GONCALVES, F.V.; RAMOS, H.M.; REIS, L.F.R. (2009). Produção de energia em sistemas de abastecimento 
baseada em fontes renováveis: Redes Neurais. In: Anais do XVIII Simpósio Brasileiro de Recursos Hídricos, Campo Grande, v. 1, p. 1-12.

GOMES, L.F.C.; MONTENEGRO, S.M.G.L.; VALE, M.J.S. (2010). Modelo baseado na técnica de redes neurais para previsão de vazões na Bacia do Rio São Francisco. Revista Brasileira de Recursos Hídricos, v. 15, n. 1, p. 05-15.

HAYKIN, S. (1994). Neural networks a comprehensive foundation. Macmillan College P. C. New York, USA.

KINOSHITA, S.K.; MARQUES, P.M.A.; FRÈRE, A.F. (2000). Utilização de Análise de Componentes Principais e Redes Neurais Artificiais para a Classificação de Nódulos em Mamogramas Digitalizados. In: Anais do XVII Congresso Brasileiro de Engenharia Biomédica, Florianópolis, 1, p. 1214-1216.

LIMA, E.B. (2012). Problemas Inversos Aplicados à Identificação de Parâmetros Hidrodinâmicos de um Modelo do Estuário do Rio Macaé. Tese (Doutorado em Modelagem Computacional), Universidade do Estado do Rio de Janeiro - UERJ/IPRJ, Nova Friburgo.

LUGON JR., J.; RODRIGUES, P.P.G.W.; SILVA NETO, A.J. (2008). Assessment of Dispersion Mechanisms in Rivers by Means of an Inverse Problem Approach. Inverse Problems in Science and Engineering, v. 16, n. 8, p. 967-979.

RODRIGUES, P.P.G.W.; GONZALEZ, Y.M.; SOUSA, E.P.; MOURA NETO, F.D. (2012a). Evaluation of dispersion parameters for River São Pedro. Brazil, by the simulated annealing method. Inverse Problems in Science \& Engineering (Print), v. 20, p.1-18.

RODRIGUES, P.P.G.W; GONZÁLEZ, Y.M.; SOUSA, E.P.; MOURA NETO, F.D. (2012b). Estimativa dos parâmetros de dispersão em rios montanhosos através do algoritmo de Luus-Jaakola. Revista Brasileira de Recursos Hídricos, v. 16, n. 5, 2012 b.

SHEN, J.; JIAB, J.; SISSONA G.M. (2006). Inverse estimation of nonpoint sources of fecal coliform for establishing allowable load for Wye River, Maryland. Water Research, v. 40, p. 3333-3342.

STRUB, I.S.; PERCELAY, J.; STACEY, M.T.; BAYEN, A.M. (2009). Inverse estimation of open boundary conditions in tidal channels. Ocean Modelling, v. 29, p. 8593.
TELLES, W.R. (2009). Simulação do transporte bidimensional horizontal de substância conservativa. Dissertação (Mestrado em Modelagem Computacional), Universidade do Estado do Rio de Janeiro - UERJ/IPRJ, Nova Friburgo.

TELLES, W.R.; SILVA NETO, A.J.; LUGON JR., J.; RODRIGUES, P.P.G.W. (2008). Simulação do comportamento de uma pluma de traçador conservativo via o método dos Volumes Finitos. In Anais do XI Encontro de Modelagem Computacional, Volta Redonda.

TONSIG, S.L. (2000). Redes Neurais Artificiais Multicamadas e o Algoritmo Backpropagation. Universidade de São Paulo, São Paulo-SP, 22 p.

YAMAMOTO, Y.; NIKIFORUK, P. (2000). A New Supervised Learning Algorithm for Multilayered and Interconnectd Neural Networks. IEEE Transactins on Neural Networks, v. 11, n. 1.

YANG, Z.; HAMRICK, J.M. (2004). Optimal control of salinity boundarycondition in a tidal model using a variational inverse method. Estuarine, Coastal and Shelf Science, v. 62, p.13-24.

\section{Simulation Of A Plume Of Contaminants In The Macae River Using Artificial Neural Networks}

\section{ABSTRACT}

The risk of a lack of drinking water is a subject of worldwide debate. Some countries already suffer from this problem due to uncontrolled water pollution by industrial and domestic waste, among others. Based on this, mathematical and computational models have been constantly used in the study of contaminant transport in river systems. They are important in the prevention and estimation of the behavior of contaminant plumes in these water bodies. This paper is a first approach to modeling the behavior of contaminants in Macae River, a major river located in the northeast part of the Brazilian state of Rio de Janeiro. It represents a starting point for studies based on Artificial Neural Networks, in lieu of a mathematical model of $2 D$ transport of pollutants. As a first approach, the behavior of a plume of contaminants is simulated for a stretch of Macae River. Experimental data are compared to the results obtained by neural networks using LevenbergMarquardt as the training method. The results presented in this work will serve as a basis for subsequent training of the neural network in order to estimate conservative substance 
Simulação de uma Pluma de Contaminantes no Rio Macaé Utilizando Redes Neurais Artificiais

concentrations at any point in a given stretch of Macae

River.

Key-words: Macae River, pollutant transport, artificial neural networks. 